\title{
CONSIDERACIONES SOBRE LA NOCIÓN DE INTUICIÓN MATEMÁTICA
}

\author{
Lina María PeÑa-Páez \\ https://orcid.org/0000-0001-7452-7014 \\ Universidad de San Buenaventura, Bogotá \\ http://dx.doi.org/10.15304/ag.39.2.6299
}

\section{Resumen}

La historia de la matemática muestra como la intuición matemática ha estado presente en la invención y desarrollo de conceptos, teorías y procedimientos matemáticos. Así mismo, ha permeado el debate filosófico, los fundamentos de la matemática y los discursos educativos; otorgándole vigencia al estudio de este tema. En el presente artículo, se exponen los argumentos bajo los cuales es posible sustentar que la intuición es un proceso, que toma ideas que se presentan, inicialmente de manera "desordenada", y que gracias al contexto y los conocimientos previos del individuo las centran en una idea "fija" que será incorporada a la matemática por la lógica y la formalización.

Palabras clave: intuición matemática, proceso, mundo real, preconceptos, formalización.

\section{Abstract}

The history of mathematics shows how mathematical intuition has been present in the invention and development of mathematical concepts, theories and procedures. Likewise, it has permeated the philosophical debate, the foundations of mathematics and educational discourses; giving validity to the study of this subject. In this article, the arguments are presented under which it is possible to support that intuition is a process, that takes ideas that are presented, initially in a "messy" way, and that thanks to the context and previous knowledge of the individual focus on a "fixed" idea that will be incorporated into mathematics by logic and formalization.

Keywords: mathematical intuition, process, real world, preconceptions, formalization.

Recibido: 24/09/2019. Aceptado: 18/02/2020. 


\section{Introducción}

La concepción de la intuición ha estado ligada a la idea de cómo el individuo adquiere el conocimiento, y en el campo particular de la filosofía de las matemáticas, está relacionada con cuestionamientos tales como: ¿el conocimiento matemático es a priori, analítico o necesario?, ¿las mentes superan a las máquinas?, ¿las matemáticas son, únicamente, operaciones mecánicas sobre símbolos? Y otro tipo de problemas, sobre la naturaleza del infinito, la verdad matemática y la existencia de los objetos abstractos, entre otros.

Caracterizar la intuición matemática, no es tarea fácil. Indagar sobre su definición lleva a una amplia y diversa gama de significados. En su libro Intuición y Razón, Bunge (1996) expone diferentes acepciones relacionadas con la intuición: totalidad, la inmediatez, la infalibilidad, evidencia e inferencia rápida. También se la puede caracterizar como "rápida, automática e inaccesible a la introspección” (Chudnoff, 2014, pp. 3-4). Otros como Fischbein (2002), en su libro Intuition in Science and Mathematics la asume como auto-evidente, cierta, intrínseca, que está relacionada con la perseverancia, coerción, estado de la teoría, extrapolación, globalidad e implicación.

Matemáticos como Kurt Gödel (2006) en su libro Obras Completas, encuentra en la intuición una función análoga a la que cumple la percepción en la física. Por su parte Kant, entendía la intuición como la facultad por medio de la cual los objetos son directamente "captados" (Kant, 1978, p. 193; A50/B74). Y finalmente, tenemos a Brouwer, quien afirmó que los objetos de la matemática son construcciones de la mente y "que nuestro conocimiento de sus propiedades fundamentales se basa en una intuición $a$ priori" (Aspray \& Kitcher, 1988, p. 7).

Ahora bien, la historia de las matemáticas ha mostrado que luego de la revolución copernicana, el auge de las geometrías no euclidianas, Cantor y el concepto de infinito real y otros hallazgos; la comunidad científica ha identificado que la intuición no siempre es evidente, ni garantía de verdad, ni mucho menos sinónimo de certeza. De hecho, "conceptos no intuitivos o contraintuitivos han invadido cada vez más la ciencia y las matemáticas"(Fischbein, 2002, p. 10).

Las grandes ideas de los talentosos matemáticos como Euler, Riemann, Cauchy o Ramanujan no surgen por el mero conocimiento de los axiomas, ellos tiene la capacidad de realizar un proceso intuitivo, que podría asemejarse al proceso mental que se requiere para armar un rompecabezas muy 
complejo, ellos pueden ver un problema desde otro punto de vista: "que una maniobra particular ayudará con la suma de una serie o la evaluación de una integral, que un problema en la teoría de números se reduce a un resultado en la teoría de funciones” (Kitcher, 1984, p. 61). Por tanto, no se espera que el matemático simplemente vea los objetos abstractos e inmediatamente pueda tener su idea fructífera, se requiere de una serie de elementos para llegar a ella.

En este artículo, cuyo objetivo es aproximarse a una noción de intuición matemática, ésta se asumirá como un proceso dinámico que no puede desconocer el contexto real del individuo (no necesariamente el físico). En principio, las ideas pueden presentarse a la conciencia de manera "desordenada", pero luego, gracias a lo ya conocido por el individuo y, en el caso de la matemática, por los conceptos y la lógica, se convierte en un acto real y comprensible para otros sujetos. Esta transformación es el resultado del proceso intuitivo llevado a cabo por el individuo. Entenderemos en este escrito que, a partir de unas imágenes aparentemente difusas dadas en la mente del matemático, la intuición será el proceso que permita convertirlas en imágenes claras y distintas, que sean aceptadas por la comunidad científica e insertadas en el sistema formal de la matemática.

El proceso de intuición experimentado por un matemático es similar al del escritor, dramaturgo, músico o poeta:

El escritor que escribe una novela, el autor dramático que crea personajes y situaciones, el músico que compone una sinfonía y el poeta que escribe una oda tienen todos en la mente algo simple y abstracto, o sea incorpóreo. Para el músico o para el poeta se trata de desarrollar en sonidos o en imágenes una impresión nueva. Para el novelista o para el dramaturgo es una tesis que hay que desarrollar en acontecimientos, un sentimiento individual o social que se ha de materializar en personajes vivientes. Se trabaja sobre un esquema de la totalidad, y el resultado se obtiene cuando se llega a una imagen distinta y clara de los elementos (Bergson, 1982, p. 178).

En el presente artículo, se exponen los argumentos bajo los cuales es posible sustentar que la intuición es un proceso, donde el mundo real y los conocimientos previos del individuo juegan un papel importante; y en el transcurso de dicho proceso, no se puede desconocer la necesidad de la lógica para formalizar los hallazgos obtenidos por la intuición.

\section{La intuición como proceso dinámico}

Asumir que la intuición es un proceso implica que, gracias a la intuición, las imágenes intangibles son convertidas en hechos reales, que pueden ser 
percibidos, como en el caso de los objetos de la física, o abstractos, como los conceptos, axiomas o proposiciones de las matemáticas. Entonces podemos afirmar que la intuición es una lucha entre el pensamiento y el mundo material, "la chispa inicial de la intuición puede desatar una reacción en cadena en el seno del conocimiento preexistente, pero el resultado final es comúnmente muy diferente de la chispa inicial” (Bunge, 1996, p.166).

$\mathrm{Al}$ ser la intuición un proceso, puede ser mejorada y entrenada. La intuición no se da de forma manifiesta en la presentación final de las teorías, tampoco deja a la lógica todo el proceso constructivo; la intuición es "un aspecto de un proceso complejo en el cual la deducción y la crítica son por lo menos tan importantes como la inspiración" (Bergson, 1982, p. 185). Cuando se realiza cualquier trabajo científico, no basta con el razonamiento inductivo y deductivo, ni con las leyes de la lógica formal. Estos deben estar acompañados de otras actividades, las cuales en su conjunto son el proceso de intuición matemática, a saber, se recurre a la búsqueda y planteamiento de problemas, se intentan controlar las soluciones encontradas, se inventan hipótesis, se elaboran deducciones y en todo este proceso intervienen la percepción de cosas, acontecimientos y signos; la imaginación o representación visual; la formación de conceptos de diverso grado de abstracción; la comparación que lleva a establecer analogías y la generalización inductiva junto con la conjetura.

Ahora bien, son poco comunes los matemáticos que pueden inventar sin la ayuda de la imaginación. Algunos "iluminados" por la intuición del número puro y de las formas lógicas no sólo pueden demostrar sino también inventar. Estos pocos analistas son capaces de percibir a simple vista el plan general de un edificio lógico, sin la intervención (aparente) de los sentidos. Sin embargo, en la mayoría de los casos "no deja de ser cierto que la intuición sensible es en matemáticas el instrumento más habitual de invención" (Poincaré, 1964, p. 9).

La invención en matemáticas como señala Poincaré está estrechamente relacionada con la intuición. Para nosotros, la invención o la creación de teorías o conceptos es el resultado de un proceso, que asumimos, como la intuición. "Quien haya hecho algo de matemáticas admitirá que su dinámica es constructiva, que el matemático no aprehende ideas platónicas preexistentes y que la axiomática es casi siempre una reconstrucción a posteriori" (Bunge, 1996, p. 102), por tanto, la intuición matemática puede asumirse como un proceso constructivo anterior al método axiomático.

Tieszen concibe la intuición como un proceso en el que se dan los objetos, y se entiende que las intenciones no están referidas exclusivamente a los 
objetos sino a los procesos que experimentan dichos objetos. Por tanto, para Tieszen (1989) el término construcción hace referencia al cumplimiento de una intención expresada en un enunciado matemático, siendo "especialmente útil para transmitir que la intuición de los objetos matemáticos debe tener lugar de manera mediatizada a través de la síntesis activa sobre la base de actos o procesos subyacentes y determinados" (p. 80). Entonces, la noción de construcción para nuestro autor es aquella en la que la intuición de los objetos matemáticos es un proceso. El término "construcción" se puede utilizar en varios sentidos. Citando a Sundholm, Tieszen (1989) encuentra que el término "construcción” puede referir a: “(i) proceso de construcción, (ii) objetos obtenidos como un resultado de un proceso de construcción, (iii) la construcción-proceso como objeto" (Tieszen, 1989, p. 86).

Desde un punto epistemológico de la intuición, se observa que ella está estrechamente relacionada con una noción ontológica de construcción, es decir, "tener intuición de X, o saber X significa que X es construible, lo que significa que $X$ existe y viceversa (Ardeshir, 2008, p. 116). Brouwer se pregunta: “¿cómo una intuición da lugar a otra intuición?” pregunta que podría ser respondida afirmando que "el acto de construcción es lo que hace el "puente" entre dos intuiciones diferentes. La razón por la que la "construcción" es una acción que no cambia la calidad intuitiva del objeto construido es que actúa en la intuición" (Ardeshir, 2008, p. 116), más aun, la exhibición de objetos matemáticos en la intuición se da gracias a la construcción.

La intuición es un fenómeno completo, dado que en ella tienen lugar: "el acto de construir objetos matemáticos en sí, 'yo' como el actor y los objetos construidos" (Ardeshir, 2008, p. 129). Entenderemos en este contexto que el proceso creativo y el proceso de intuición están relacionados "eso significa que la intuición sin construcción está vacía y no es una percepción en absoluto, y la construcción es una acción solo en la intuición" (Ardeshir, 2008, p. 116). Ahora bien, esta relación intuición-creación y el hecho de que los dos procesos se dan en la conciencia, nos permite comprender que "cualquier juicio o proposición matemática es algún tipo de informe de lo que ha sido construido en mi intuición" (p. 129).

La intuición matemática es ese proceso, que involucra varias capacidades del individuo, por medio del cual, y partiendo del contexto, construye nuevos conceptos o teorías. En el siguiente apartado se explica el contexto real del individuo. 


\section{El papel del mundo real del individuo en la intuición matemática}

De acuerdo con Popper, existen tres mundos. El mundo de los cuerpos físicos, el de los estados de conciencia y el mundo de los productos de la mente humana. En este tercer mundo encontramos los conceptos y teorías matemáticas. En cualquier caso, ya sea para inventar una nueva teoría, para determinar un concepto o simplemente para resolver un problema, el matemático toma como base su mundo real (que puede ser cualquiera de los tres mencionados).

Popper nos explica en qué sentido los objetos del mundo 3 son reales:

1. Los objetos del mundo 3 son reales (aún más abstractos que las fuerzas físicas), pero aún así, son reales, pues constituyen herramientas poderosas para cambiar el mundo 1. (No pretendo dar a entender que esa sea la única razón para considerarlos reales, ni que sean simplemente herramientas).

2. Los objetos del Mundo 3 poseen efectos sobre el Mundo 1 sólo a través de la intervención humana de sus creadores; más concretamente, poseen dichos efectos gracias a que son capatados, lo que constituye un proceso del Mundo 2, más exactamente, un proceso en el que entran en interacción los Mundos 1 y 3.

3. Por tanto, hemos de admitir la realidad tanto de los objetos del Mundo 3 como de los procesos del Mundo 2, aun cuando pueda no gustarnos admitirlo por deferencia, digamos, hacia la gran tradición del materialismo. (Popper \& Eccles, 1977, p. 54)

Bajo este marco, los resultados de la intuición matemática son producto de la interacción de los elementos que "habitan" cada uno de esos mundos. Durante el proceso los elementos de estos tres mundos se organizan para encontrar un resultado. Inicialmente las ideas sin "orden" aparente son introducidas y "mezcladas" con las ideas antiguas para luego ser comunicadas y aceptadas gracias a la intervención de los sistemas formales de la matemática.

En el mundo de los objetos corporales que rodean al individuo, "la información adquirida debe convertirse en representaciones de la realidad aparentemente bien estructuradas, autoconsistentes y orientadas a la acción"(Fischbein, 2002, p. 15). Ahora bien, los individuos también pueden obtener información sin tener un contacto directo con los objetos, por ejemplo, a través del lenguaje, el razonamiento y la matemática. De acuerdo con Popper podemos afirmar que los objetos, los procedimientos, las definiciones y reglas establecidas formalmente en la matemática "habitan" el tercer mundo.

La naturaleza de los objetos de cada uno de estos dos mundos es diferente como lo expresa Fischbein (2002, p. 15): 
Las cualidades adaptativas habituales de las representaciones objetivas: inmediatez, evidencia propia, autoconsistencia, credibilidad directa, necesidad intrínseca (como aparecen en las percepciones sensoriales) están ausentes de las entidades matemáticas. En lugar de la credibilidad intrínseca que ofrecen los objetos reales y las operaciones prácticamente realizadas, las matemáticas tratan con un tipo de certeza basado formalmente. En lugar de objetos concretos, las matemáticas postulan formalmente la existencia de entidades abstractas. En lugar de la verificación empírica, las matemáticas utilizan controles deductivos a través de pruebas formales. La evidencia probada reemplaza a la evidencia directa. En lugar de la coherencia intrínseca, dada, de las realidades empíricas, las matemáticas se esfuerzan por crear conjuntos de oraciones, cuya coherencia y consistencia se establecen formalmente. Este es un mundo nuevo, fundamentalmente diferente del de los objetos reales y los eventos reales: el mundo de las construcciones mentales, regido internamente por leyes formalmente establecidas, el mundo de las matemáticas. Se pretende que funcione de una manera absolutamente autárquica: produce sus propios objetos; los relaciona uno con el otro según sus propios principios; tiene su tipo específico de necesidad: necesidad lógica en lugar de causalidad empírica; tiene su propio tipo de certidumbre, un tipo de certidumbre que se puede reducir al rigor formal (y que puede no tener ninguna relevancia práctica).

La descripción anterior parece sugerir que el mundo de la matemática es absolutamente independiente de los otros dos mundos en los que el individuo se mueve, es un mundo cerrado de proposiciones meramente formales. Pero esta absoluta independencia ha mostrado ser imposible. Gödel y su teorema de incompletitud dejó en evidencia que "un sistema matemático nunca puede cerrarse por completo; es decir, nunca puede poseer en sí todos los prerrequisitos formales necesarios para decidir sobre la validez de todos sus teoremas" (Fischbein, 2002, p. 16).

Tenemos, entonces, que la intuición está relacionada con esos tres mundos de los que habla Popper. En el mundo físico, por ejemplo, no existe la línea recta, ésta es una abstracción. Existen interpretaciones diversas como haz de luz, como una línea dibujada en un papel, como caminar recto. Ahora bien, cuando una persona usa el término "línea recta", independiente de su análisis teórico, sabe de lo que está hablando. Sin duda, el concepto de línea recta no tiene un significado absoluto, tiene un significado en la práctica. Este término es una convención definida bajo un grupo de postulados, que podría cambiar, si se cambia el sistema de referencia o los axiomas.

El matemático en su proceso intuitivo requiere de la experiencia que le brindan estos tres mundos. Algunas descripciones empíricas y algunos análisis teóricos muestran que la experiencia acumulada de un individuo es una fuente para la intuición. Fishbein $(2002$, p. 85) expone tres aspectos experimentales que son relevantes para la intuición:

(a) Los elementos generales y comunes de la experiencia humana. 
(b) Los aspectos de la experiencia relacionados con el entorno geográfico y cultural particular en el que vive una persona.

(c) La práctica particular del individuo relacionada con varios dominios de su vida (por ejemplo, intuiciones profesionales).

En suma, no es posible pensar la intuición matemática alejada de la experiencia del individuo en su realidad, dado que es él quien advierte la intuición. Los resultados del proceso intuitivo se dan gracias a la experiencia tanto en la matemática como en la vida cotidiana, "la experiencia, entonces, juega un papel fundamental en dar forma a nuestras intuiciones y esto, como he dicho, explica, al menos parcialmente, su impacto en cualquier esfuerzo productivo, teórico o práctico"(Fischbein, 2002, p. 88).

\section{La intuición y las ideas previas}

En este apartado nos interesa abordar la relación entre la intuición matemática y las ideas o experiencias previas fijadas, que no necesariamente son conscientes. Algunos como Kitcher, desde un punto de vista del naturalismo, consideran que, "los axiomas de una teoría no son sus comienzos. Más bien son el resultado de una sistematización de un 'cuerpo previo de afirmaciones' sobre los objetos de la teoría que han sido empleados tácita o explícitamente en el razonamiento"(Sierpinska \& Lerman, 1996, p. 836).

Es gracias a la intuición que el individuo puede transformar, comprender y dar significado a las relaciones entre los elementos de un conjunto. Pero esto sólo será posible si se tiene un entrenamiento previo: "el principiante puede 'intuir' ciertos objetos, pero el iniciado captará además ciertas relaciones y complejidades que escaparán al novato" (Bunge, 1996, p. 123). Por tanto, la intuición creadora, la que permite hablar de invención y de inspiración, aparece cuando se engendran nuevas ideas, sin aparente esfuerzo ni lógica explícita. Esta capacidad de invención y construcción, permite que el trabajo del matemático vaya más allá de lo algorítmico: "la invención de hipótesis y técnicas, y el diseño de experimentos son casos patentes de operaciones imaginativas o, si se prefiere, de actos intuitivos" (Bunge, 1996, p. 138). Esta situación no implica que las ideas novedosas surjan de la nada, es necesario que el individuo tenga cierto tipo de conocimiento previo. Los nuevos conocimientos serán "adquiridos” gracias a la observación, la comparación, la deducción, en general, gracias a una combinación entre la intuición y la formalización matemática. 
Existen muchas formas de obtener información, a través de herramientas, instrumentos, lenguaje, lógica y razonamiento, dicha información gracias a la intuición se convierte en una representación estructurada, coherente y orientada al mundo de los objetos y eventos que nos rodean. Podríamos afirmar que el "éxito" de una intuición sensible (aprehensión de un objeto físico) dependerá de la habilidad perceptual del sujeto, de su memoria, de su inteligencia, de su experiencia y de la información de la que disponga, es decir, "la base de las creencias funcionará para hacer que nosotros intuyamos más determinados” (Tieszen, 1989, p. 5).

En la creación o invención de alguna idea matemática, encontramos que el individúo no sólo debe contar con unos conocimientos matemáticos previos, sino con herramientas lingüísticas adecuadas para comprender conceptos como triángulo, objeto físico y conjunto. Para Maddy, por ejemplo, un niño de tres años es capaz de percibir objetos físicos, pero no tiene las capacidades lingüísticas para expresarse como las tendría un matemático profesional. Los matemáticos con sus herramientas lingüísticas y el conocimiento de teorías matemáticas parecen verse forzados a aceptar enunciados matemáticos como si fueran verdaderos, y la razón es que dichos enunciados han sido formados por las diversas creencias intuitivas. De ahí que los principios intuitivos se entiendan como los "intentos de formular creencias intuitivas en términos lingüísticos" (Maddy, 1980, p. 186).

Un individuo, gracias a la intuición, puede transformar una idea en hechos concretos, por ejemplo, enunciados matemáticos y, por medio del lenguaje, comunicarla a la comunidad científica. Pero el proceso de intuición no es solamente "desarrollado" por los matemáticos, también lo podríamos evidenciar cuando un profesor o un estudiante quieren resolver un problema. Quien se enfrenta a un problema matemático, tiene una serie de conocimientos anteriores y la reagrupación de estas ideas previas, la organización de las mismas, que son etapas de la intuición, le permitirán resolver el problema planteado.

Las ideas ganan en precisión y claridad a medida que el individuo desarrolla más capacidades formales, por ejemplo, quien "aprende sobre la naturaleza de los números racionales y reales puede obtener una comprensión más nítida y clara del concepto de número" (Fischbein, 2002, p. 202), aunque la idea de número permanezca unida a las ideas de cardinalidad y orden, propias de los números naturales. Las nuevas creencias cognitivas surgen bajo la influencia sistemática de las antiguas intuiciones, "tal proceso implica, en nuestra opinión, la participación personal del aprendiz en una actividad en la que las cogniciones respectivas desempeñan el 
papel de representaciones necesarias, anticipatorias y, luego, confirmadas" (Fischbein, 2002, p. 202).

No podemos olvidar que "las intuiciones son siempre el producto de la experiencia personal, de la participación personal del individuo en una determinada actividad práctica o teórica" (Fischbein, 2002, p. 213). Sólo quien esté inmerso en situaciones que involucren conjeturas, predicciones, solución de problemas, teorías matemáticas, proposiciones lógicas, es decir, todo un contexto científico y matemático tendrá la posibilidad de "intuir" o inventar nuevas teorías o conceptos. La historia nos ha mostrado, por ejemplo, que las más novedosas ideas matemáticas han sido elaboradas por matemáticos y no por personas ajenas a la disciplina.

\section{La intuición y su necesidad de convalidación}

En este último apartado se analiza la necesidad de que las ideas, conjeturas, conceptos o teorías conseguidos gracias a la intuición deban pasar por el "filtro" de la matemática formal. El proceso intuitivo que lleva a un matemático a sus ideas geniales o los conceptos o teorías, debe ser validado por una demostración rigurosa, o en su defecto, por los resultados verdaderos de la aplicación de dicha idea.

La historia de la matemática nos trae un ejemplo: Ramanujan, quien no había tenido ayuda de nadie, simplemente se había encontrado un libro de aritmética, lo estudió y llegó donde llegó, pero él no sabía hacer demostraciones, su intuición le mostraba que las cosas eran así. Por tanto, cuando llega a Cambridge, no le cabe en la cabeza la idea de la demostración, y, sin embargo, sus facultades se habían desarrollado en contacto con algo, que en último término era lingüístico: un libro. Resulta sorprendente ver cómo la mente humana es capaz de crear cosas, que no necesariamente tienen su origen en el mundo físico, pero sí en el mundo de la matemática. Estas teorías que han sido el resultado de un proceso intuitivo, se puede ir haciendo cada vez más abstractas y desarrolladas, hasta que en el algún punto alguien podría encontrar una aplicación en el mundo físico, siempre y cuando hayan sido convalidadas. De ahí, que la matemática sea ese lenguaje potente que tenemos para entender y explicar el mundo en el que vivimos.

Ahora bien, conocer todos los símbolos de la matemática, sus axiomas, teoremas o reglas, por sí solos no nos llevarán a las grandes ideas o creaciones matemáticas, se requiere algo adicional, "la relación entre ciertas construcciones (símbolos, conceptos, proposiciones) y las correspondientes 
experiencias sensoriales no es lógica sino “intuitiva”, como lo señaló Einstein" (Bunge, 1996, p. 127). Los sistemas formales o las aplicaciones efectivas serán las que permitan validar lo que la intuición nos brinda de primera mano.

La intuición dará frutos en la medida que sea perfeccionada por la razón. Una vez en el pensamiento del matemático, la idea obtenida por el proceso intuitivo, es validada por las teorías matemáticas existentes, ésta se incorpora a un cuerpo de proposiciones lógicas y se enlazan para nuevas construcciones conceptuales. La intuición matemática es anterior a la sistematización y a la etapa de formalización, por lo que no debe considerarse como un modo extraordinario de conocer o como "cierto tipo de revelación independiente"(Bunge, 1996, p. 50). Las leyes son construcciones, las cuales se convertirán en "esenciales" cuando hayan pasado las pruebas lógicas y matemáticas.

En las ciencias empíricas se recurre a la heurística y a la inducción para resolver problemas. Para Fischbein: "la idea principal es que el mismo tipo de actitudes y esfuerzos mentales que caracterizan un intento empírico de solución también intervienen a nivel formal" (2002, p. 23). En la matemática se recurre "a las formas intuitivas de aceptación y extrapolación que pueden asegurar su firmeza de comportamiento requerida, su productividad, su consistencia dinámica y flexible" (2002, p. 23).

Para Maddy las creencias intuitivas pueden ser equivocadas, por lo que afirma que es necesario: 1. Que la intuición sea compartida por otros y 2. Que los enunciados que se refieran a las creencias intuitivas se sustenten en teorías. Las condiciones necesarias para que un enunciado se acepte como sustento extrínseco son (Stisman, 2006, p. 536):
a) Que las consecuencias del enunciado sean susceptibles de verificación.
b) Que puedan resolver problemas ya existentes.
c) Que la teoría se simplifique y sistematice.
d) Que conlleve conjeturas previas
e) Que sus resultados sean naturales
f) Que sus conexiones inter-teóricas con otros resultados sea fuertes.
g) Que brinde a los teoremas antiguos nuevos ambientes de aplicación.

Un buen ejemplo de combinación entre la intuición y las consideraciones teóricas es el axioma de elección. Zermelo afirmó que muchos matemáticos hicieron uso inconsciente del axioma, aunque luego lo negaran. Este es el carácter intuitivo del axioma. De otra parte, el hecho de que la teoría de conjuntos fundamente la aritmética implica un apoyo teórico o extrínseco. Para sustentar el carácter teórico del axioma se nombran los siguientes 
hechos: "1) Permite deducir de forma más simple otros enunciados derivados de su auxilio. 2) Permitió resolver problemas abiertos como el del buen orden. 3) Simplifica y unifica la teoría de conjuntos al ordenar la aritmética transfinita" (Stisman, 2006, p. 536).

En algunos casos, las afirmaciones matemáticas que se han utilizado no tienen demostraciones rigurosas o aún no se han demostrado, lo que no significa que sean falsas; tienen una utilidad inductiva y explicativa. De esta forma, podríamos entender el proceso intuitivo como "una prueba amable, pero solo sobre algo muy específico y limitado, ya que no hacen nada más que hacer explícito el contenido de los conceptos matemáticos implicados en las intuiciones relevantes" (Fedyk, 2018, p. 96). No necesariamente el resultado de la intuición genera una verdad exacta: "la razón de esto es que el concepto implicado ha sido moldeado por cualquier proceso que lo lleve a su hogar inferencial en el desordenado mundo del razonamiento científico" (Fedyk, 2018, p. 103). Ahora bien, las creencias que se tienen en la ciencia, se fundamentan en los conceptos que a su vez son el resultado de la intuición: "de acuerdo con esta teoría, las intuiciones son procesalmente instrumentales para la formulación de creencias matemáticas" (Fedyk, 2018, p. 103).

Como se ha señalado, en la historia de la matemática ha sido necesario, en algunas ocasiones, controlar la carga intuitiva y su impacto en el razonamiento de conceptos, dado que en las matemáticas, el conflicto principal aparece entre las tendencias intuitivas de la actividad de razonar y la naturaleza puramente formal y abstracta de los conceptos, por lo que se hace necesario, "tomar conciencia del significado exacto y formal y las implicaciones de los conceptos matemáticos, por un lado, y las intuiciones subyacentes, por el otro" (Fischbein, 2002, p. 207).

La discusión central, ya no es si deben o no aceptarse las intuiciones en matemáticas o si estas son un tipo de representación incompleta y parcial, sino que se debería intentar comprender que la intuición que permitió llegar a un concepto o teoría da paso a nuevas estructuras con una estructura lógica propia, expresada en una mayor coherencia interna, que, por su naturaleza formal, será adaptada a la teoría ya existente y redundará en nuevos avances en la matemática y en la ciencia en general. 


\section{Conclusiones}

Delimitar la intuición matemática en un concepto completamente estructurado, no es tarea fácil. La literatura al respecto muestra que la noción de intuición lleva consigo influencia de diferentes tipos: creencias matemáticas, lecturas previas, épocas de la historia resultados de proyectos de investigación. En lo que muchos de los estudios coinciden, es en el papel protagónico que la intuición ha jugado, tanto en la matemática pura, al contribuir en el desarrollo de grandes teorías o procedimientos; como en la filosofía de las matemáticas, al intervenir en temas como la naturaleza de los objetos y en la epistemología de las matemáticas.

Al revisar las diferentes concepciones sobre la noción de la intuición matemática, tomamos elementos comunes de los autores estudiados y entre ellos encontramos que la intuición es un proceso dinámico, que está relacionado con la experiencia, con los conocimientos matemáticos del individuo que intuye y finalmente, que requiere de las matemáticas formales para validar sus creaciones.

La intuición es el resultado de un proceso que requirió de mucho esfuerzo, de ensayar diversas hipótesis, de deducir consecuencias y de compararlas con los datos empíricos; los resultados deben ser puestos a prueba para hacerlos compatibles con el conjunto del conocimiento científico. La intuición es un esfuerzo intelectual, es decir, el que va más allá de la percepción de los sentidos o de la simple memoria. La intuición podría verse como el punto final de un trabajo previo que implica prueba, ensayo y error, indeterminación, introspección, interpretación, análisis, memoria etc., pero a la vez, es el punto de partida del trabajo lógico y formal de la matemática. La intuición indeterminada, es una construcción de la mente humana, en general, no es una revelación divina.

La intuición matemática surge cuando el individuo ha estado inmerso en un contexto matemático. Cuanto mayor sea su conocimiento sobre la matemática, mejor será su proceso intuitivo, sin embargo, en este proceso se requiere más que sólo los datos disponibles, va más allá de cualquier conclusión a la que una persona hubiese llegado conociendo únicamente estos datos. Elaborar una conjetura o una hipótesis, es producto de un interés por un problema particular. Por tanto, aunque se tengan los axiomas, las herramientas adecuadas o las leyes de la lógica, estos insumos no bastan para llegar a conjeturar enunciados o proposiciones.

La intuición surge en un contexto histórico o físico, y este contexto es el mundo real del individuo que intuye. Un científico que invente una 
teoría novedosa está inmerso en un ambiente que le proporciona los insumos para tener ese momento de "inspiración". Las ideas no surgen de la nada. No se puede pretender que una persona que nunca ha realizado un cálculo matemático invente un concepto. "Por ejemplo, alguien que no tiene ningún concepto de quarks en absoluto, no tendrá intuiciones diseñadas para probar si alguna nueva partícula hipotética puede contar como el séptimo quark" (Fedyk, 2018, p. 92).

En el proceso de intuición, las ideas sobre conceptos abstractos, que inicialmente pueden "aparecer" de manera "desordenada", se van organizando junto con las ideas antiguas que el individuo ya tiene, para de esta forma crear sus nuevos conceptos o enunciados. Este proceso no será fructífero si el individuo no maneja de manera clara los conceptos matemáticos, de lo que se infiere que, el conocimiento de lo nuevo es posible cuando el individuo se apoya en lo ya conocido. Cuando un individuo intenta resolver un problema, plantear una hipótesis o verificar una solución, requiere de un conocimiento formal previo en su campo de estudio, es decir, que debe contar con cierto tipo de información confiable, de tal forma que el proceso intuitivo, le permita articular sus ideas en una estructura lógica susceptible de verificación por los mecanismos de la matemática formal.

La validación de los resultados obtenidos por la intuición matemática, deben cumplir con los requerimientos de la matemática formal, con sus axiomas, teoremas y procedimientos. Cuando los matemáticos están interesados en elaborar nuevas teorías, recurren a diversos mecanismos para lograr su objetivo: asimilación de conocimientos previos, imaginación, ensayo de hipótesis, síntesis para resolver un problema o validación de las conjeturas planteadas, y en este proceso aparece la intuición. Muchas de las creencias de los individuos surgen de un proceso de intuición, la validez o no de las mismas requiere la intervención de procedimientos formales, lo que implica que tanto la intuición como la formalización son necesarias para el conocimiento de las matemáticas.

Este trabajo pretende plantear una postura frente a la intuición, sin embargo, quedan muchos interrogantes al respecto, por ejemplo: ¿cuál es el papel de la intuición en el conjunto de creencias de matemáticos y estudiantes?; ¿si la intuición es un proceso, ¿cómo puede éste fortalecerse para que produzca más ideas novedosas?; ¿puede establecerse una relación entre la cantidad de preconceptos estructurados del individuo y el resultado de sus intuiciones? 


\section{Bibliografía}

Ardeshir, M. (2008). Brouwer's notion of intuition and theory of knowledge by presence. En M. van Atten, P. Boldini, M. Bourdeau, \& G. Heinzmann (Eds.), One Hundred Years of Intuitionism (1907-2007). The Cerisy Conference Basel, Birkhäuser, 2008, pp. 115-130. https://doi. org/10.1007/978-3-7643-8653-5 8

Aspray, W. \& Kitcher, P. History and Philosophy of Modern Mathematics, Minneapolis, University of Minnesota Press, 1988.

Bergson, H. La Energía Espiritual, Madrid, Espasa Calpe, 1982.

Bunge, M. Intuición y razón, Buenos Aires, Editorial Sudamericana, 1996. Chudnoff, E. "Intuition in Mathematics", en L. M. Osbeck \& B. S. Held (Eds.), Rational Intuition: Philosophical Roots, Scientific Investigations, Cambridge, Cambridge University Press, 2014, pp. 174-191. https://doi. org/10.1017/CBO9781139136419.010

Fedyk, M. "Intuitions, Naturalism, and Benacerraf's Problem", en S. Bangu (Ed.), Naturalizing Logico-Mathematical Knowledge, New York, Routledge, 2018, pp. 89-105. https://doi.org/10.4324/9781315277134-5

Fischbein, E. Intuition in Science and Mathematics. An Educational Approach, Dordrecht, Reidel, 2002.

Gödel, K. Obras Completas, Madrid, Alianza editorial, 2006.

Kant, I. Crítica de la Razón Pura, Madrid, Alfaguara, 1978.

Kitcher, P. The Nature of Mathematical Knowledge, New York, Oxford University Press, Inc., 1984.

Maddy, P. "Perception and Mathematical Intuition", The Philosophical Review, (1980), 163-196. https://doi.org/10.2307/2184647

Poincaré, H. "La intuición y la lógica en las Matemáticas". En El valor de la ciencia, 1905, pp. 1-9. Recuperado de http://casanchi.com/ref/logicaintuicion01.pdf 1905.

Popper, K. \& Eccles, J. El yo y su cerebro, Barcelona, Editorial Labor, 1977. Sierpinska, A. \& Lerman, S. "Epistemologies of mathematics and of mathematics education", en A. J. Bishop, K. Clements, C. Keitel, J. Kilpatrick, \& C. Laborde (Eds.), International Handbook of Mathematics Education, Dordrecht, HL: Kluwer, 1996, pp. 827-876.

Stisman, A. F. "El realismo conjuntista de Penelope Maddy", Epistemología e historia de la ciencia, (2006), 533-539.

Tieszen, R. Mathematical intuition: phenomenology and mathematical knowledge, Dordrecht, Kluwer Academic Publishers, 1989.

https://doi.org/10.1007/978-94-009-2293-8 